\title{
Author Correction: Broadband and pixelated camouflage in inflating chiral nematic liquid crystalline elastomers
}

Se-Um Kim, Young-Joo Lee, Jiaqi Liu (1D, Dae Seok Kim, Haihuan Wang and Shu Yang (1)

Correction to: Nature Materials https://doi.org/10.1038/s41563-021-01075-3, published online 6 September 2021

In the version of this Letter initially published, there was an omission in Fig. 2. Specifically, in Fig. 2a, bottom left, the structure "5CB" originally showed a single phenyl ring. The structure has now been amended to show both phenyl rings.

These changes have been made to the print and online versions of the Letter.

Published online: 27 September 2021

https://doi.org/10.1038/s41563-021-01140-x

(C) The Author(s), under exclusive licence to Springer Nature Limited 2021 